\title{
Uso de Tecnologias Digitais Sociais no Processo Colaborativo de Ensino e Aprendizagem
}

\author{
André Barros de Sales ${ }^{1}$, Clodis Boscarioli ${ }^{2}$ \\ andrebdes@unb.br, clodis.boscarioli@unioeste.br \\ ${ }^{1}$ Universidade de Brasília (UnB), Faculdade do Gama, Brasília, DF, 72.444-240, Brasil \\ ${ }^{2}$ Universidade Estadual do Oeste do Paraná (Unioeste), campus de Cascavel, PR, 85819-110, Brasil
}

DOI: 10.17013/risti.37.82-98

\begin{abstract}
Resumo: As tecnologias digitais, em especial as sociais, cada vez mais presentes no cotidiano das pessoas em todo o mundo, e são utilizadas em diferentes contextos e com diferentes finalidades, além de promover os processos de ensino e aprendizagem entre os estudantes, principalmente no ensino superior. Este artigo traz uma análise do uso de tecnologias sociais à luz da visão dos estudantes universitários numa disciplina do curso de Engenharia de Software da Faculdade UnB Gama da Universidade de Brasília, de como essas tecnologias contribuem para a sua aprendizagem, em aulas ministradas com metodologias ativas. Além das preferências por ferramentas, os dados coletados indicam seu potencial no aprendizado personalizado e coletivo, na sistematização dos trabalhos colaborativos, bem como no suporte e no gerenciamento das tarefas para realizar os projetos propostos.
\end{abstract}

Palavras-chave: Processos colaborativos de Ensino e Aprendizagem; Tecnologias digitais sociais; Tecnologias de Comunicação e Informação na Aprendizagem.

\section{Using Social Digital Technologies in the Teaching and Learning Process in Higher Education}

\begin{abstract}
Digital technologies, especially social technologies, are increasingly present in the daily lives of people around the world, and are used in different contexts and with different purposes, in addition to promoting teaching and learning processes among students, especially in higher education. . This article presents an analysis of the use of social technologies in the light of the view of university students in a discipline of the Software Engineering course at University of Brasília, of how these technologies contribute to their learning, in classes taught with active methodologies. In addition to preferences for tools, the data collected indicates their potential for personalized and collective learning, for systematizing collaborative work, as well as for supporting and managing tasks to carry out the proposed projects.
\end{abstract}

Keywords: Collaborative teaching and learning processes; Social digital technologies; Communication and Information Technologies in Learning. 


\section{Introdução}

Os celulares são dispositivos cada vez mais utilizados por todos os estratos sociais e todas as faixas etárias. Nas últimas décadas, os celulares tradicionais básicos vêm sendo substituídos por smartphones, celulares com conexão à internet e aplicativos com inúmeras possibilidades de uso para fins sociais, e tem muito potencial para uso em situações de aprendizado colaborativo.

Assume-se aqui que tecnologias digitais sociais são as tecnologias digitais que permitem interação social e práticas colaborativas, como aquelas em que os estudantes escolham o controle de seu próprio aprendizado, mediado por uma série de ferramentas, incluindo áudio, vídeo, gerenciamento de grupos e armazenamento e compartilhamento de dados. Para Chagas et al. (2019), as instituições de ensino superior devem preparar-se para dotar seus estudantes de conhecimentos que lhes possibilitem desenvolver habilidades e competências para trabalhar de forma colaborativa nos ambientes digitais. Wander et al. (2012) considera habilidades como "capacidades tanto físicas, quanto intelectuais, e que estão relacionadas ao saber fazer" e competências como "capacidade de articular e mobilizar conhecimentos, habilidades e atitudes, colocando-os em ação para resolver problemas e enfrentar situações de imprevisibilidade em uma dada situação concreta de trabalho e em um determinado contexto cultural", definições essas adotadas na condução da pesquisa ora relatada.

Muitas dessas tecnologias não foram construídas para o processo de ensino e aprendizagem, mas para terem boa usabilidade onde quer que se apliquem. Nas instituições de ensino superior em todo o mundo, há uma infinidade de experiências em pequena escala e de integração de uma variedade de recursos de software social em práticas pedagógicas. Dada essa diversidade no ensino superior, é enorme o conhecimento dos discentes sobre uma variedade desses aplicativos, software e tecnologias num curso de exatas na área de computação.

O avanço na área de desenvolvimento de software pode ser significativamente influenciado pela qualidade da educação no ensino de Engenharia de Software - ES, que auxilia na solução de alguns problemas tradicionais com as práticas da indústria de software (Gibbs, 1994), e pelos resultados encontrados. Lima et al. (2019) mostram que as metodologias ativas promovem aplicação de atividades que envolvem práticas do mundo real, elevação das competências interpessoais e a motivação dos discentes quando se deparam com as questões pedagógicas presentes.

A disciplina de Interação Humano-Computador (IHC) busca melhorar a experiência de pessoas com os dispositivos eletrônicos, de maneira a torná-la mais produtiva e eficiente e tornar o uso desses dispositivos mais agradável e intuitivo. IHC é uma disciplina que demanda uma atenção especial aos conceitos, e por ser mais teórica, torna-se difícil despertar o interesse dos discentes pelo conteúdo e de desenvolver neles as competências profissionais desejadas no mercado. O conteúdo da disciplina e sua aplicação apresenta a necessidade de oferecer, aos discentes, diferentes abordagens e recursos didáticos que os envolva no processo de ensino e aprendizagem, como as descritas em (Júnior, 2010; Cerutti, 2010; Sales et al, 2013). 
No processo de ensino e aprendizagem, diversas abordagens de metodologias InovAtivas (Ativas, Ágeis, Imersivas e Analíticas) têm sido pesquisadas e usadas (Filatro e Calvacanti, 2018). Uma dessas abordagens é a Aprendizagem baseada em Projetos (PBL - Projects Based Learning), que permite aos estudantes articulação com a realidade profissional, unindo teoria e prática. Nessa abordagem os discentes também podem desenvolver competências transversais, como: atividades colaborativas; condução de reuniões; tomada de decisão e gerenciamento de conflitos e projetos (Correia e Oliveira, 2020; Filatro e Calvacanti, 2018; Santana, 2009, Fernández, 2006).

Por meio do Programa Aprendizagem para o $3^{\circ}$ Milênio (A3M), a Universidade de Brasília (UnB) tem apoiado a pesquisa, a produção de novos conhecimentos e o desenvolvimento de metodologias educacionais inovadoras no processo de ensino e aprendizagem. Oriundo de tal contexto, este artigo apresenta as principais tecnologias digitais sociais e a facilidade de seu uso quando utilizadas no processo de ensino e aprendizagem por discentes numa turma que emprega a abordagem Aprendizagem baseada em Projetos na disciplina de Interação Humano-Computador do curso de Engenharia de Software da Faculdade do Gama da Universidade de Brasília.

Este artigo segue assim organizado: a Seção 2 apresenta o embasamento teórico da pesquisa; a Seção 3 contempla a metodologia; na Seção 4 discutem-se os resultados, e na Seção 5 são tecidas as considerações finais.

\section{Referencial Teórico}

Os diversos software da Web 2.0, como blogs, compartilhamento de mídia ponto a ponto (P2P) e wiki estão oferecendo oportunidades de aprendizado sem precedentes. Em todo o mundo, os estudantes são capazes de usar a inteligência coletiva para criar "a sabedoria das multidões" (Surowiecki, 2005), conectando-se a ambientes sociais ricos e dinâmicos, ao invés de estudarem sozinhos. McLoughlin et al. (2007) definem a Pedagogia 2.o como a combinação das possibilidades das tecnologias sociais e abordagens educacionais, que oferecem um potencial de mudanças radicais e transformacionais nas práticas de ensino e aprendizagem com ferramentas que resultarão em ambientes de aprendizado mais personalizado, participativo e colaborativo.

O computador, principalmente por meio da internet, tem possibilitado a aprendizagem colaborativa como abordagem pedagógica em que a aprendizagem ocorre por meio da interação social virtual. Segundo (Stahl, Koschmann e Suthers, 2006) esse tipo de aprendizado é caracterizado pelo compartilhamento e construção de conhecimento entre os participantes, usando a tecnologia como principal meio de comunicação ou como um recurso comum.

Nesse contexto, as metodologias ativas se aplicam como práticas pedagógicas que levam o estudante a fazer e a pensar no que está fazendo à luz dos conceitos teóricos circunstanciados em práticas do seu próprio cotidiano. Nessa metodologia, o estudante é envolvido em seu processo de aprendizado como protagonista de sua formação, da construção do seu próprio conhecimento, habilidade e competência, como nas seguintes práticas de ensino e aprendizagem nas metodologias ativas de aprendizagem: 
aprendizagem baseada em problemas; estudo de caso, aprendizagem entre pares e aprendizagem baseada em projetos, utilizada nesta pesquisa.

De acordo com Bender (2014), a PBL é "um modelo de ensino que consiste em permitir que os estudantes confrontem as questões e os problemas do mundo real que consideram significativos, determinando como abordá-los, e agindo de forma cooperativa em busca de soluções".

Escolhemos a abordagem baseada em projetos porque nela os estudantes precisam se esforçar, individual e coletivamente, para explorar as possíveis soluções de um contexto específico, para o qual devem ser utilizadas as tecnologias ou recursos disponíveis, o que incentiva o desenvolvimento de um perfil investigativo e crítico ante uma situaçãoproblema. Além disso, o professor presente, além de expor a metodologia a ser trabalhada para que os estudantes busquem os conhecimentos por si mesmos, atua como tutor fornecendo orientações e provendo feedback nos projetos em desenvolvimento, mostrando erros e acertos da trajetória de construção coletiva do conhecimento.

Para Blumenfeld et al. (1991), a aprendizagem baseada em projetos difere da aprendizagem baseada em problemas porque nesta última os estudantes são focados principalmente no processo de aprendizagem, enquanto na primeira, para além disso, precisaria culminar num produto. Segundo Wrigley (1998), a maioria das práticas de aprendizagem baseadas em projetos abrange as seguintes etapas: seleção de tópicos, planejamento, pesquisa e elaboração de produtos.

Quando se desenvolve um projeto, os estudantes e o professor podem perder o foco dos objetivos de aprendizagem estabelecidos e não esgotar os conteúdos curriculares propostos. Esse é um dos problemas da abordagem Aprendizagem baseada em Projetos. Mesmo que o protagonismo dos discentes nessas situações de aprendizado seja mais acentuado, é imprescindível acompanhar cada etapa do projeto, cabendo ao professor oferecer-lhes ajuda e orientação durante todo essa trajetória (Filatro e Calvacanti, 2018).

O Projeto Pedagógico do Curso - PPC de Engenharia de Software da Faculdade do Gama (FGA), em sua primeira versão, fundamentou-se principalmente nas diretrizes do Software Engineering 2004 (IEEE-CS/ACM), Conselho Nacional de Educação CNE/CES No. 11, de 11/03/2002, Currículo de Referência da Sociedade Brasileira de Computação (SBC 2005) e Modelos de Processos de Software (CMMI e MPS. BR) (Figueiredo et al., 2010). Convém salientar que a segunda versão do PPC de Engenharia de Software da FGA, em outubro de 2016, seguiu a Resolução no. 5/2016 (Resolução CNE/CES, 2016) na orientação da formação profissional por habilidades e competências (Wander et al., 2012).

\section{Metodologia}

"Esta pesquisa foi desenvolvida após três meses do início do semestre, com $75 \%$ das aulas já ministradas, em caráter exploratório, que segundo (Gil, 1999), é aquela feita “com o objetivo de proporcionar uma visão geral, de tipo aproximativo, acerca de determinado fato"." A abordagem é do tipo qualitativa, e o grupo social analisado se constituiu de discentes da disciplina Interação Humano-Computador (IHC) do curso de graduação em Engenharia de Software da Faculdade do Gama da Universidade de Brasília, Brasil, 
no segundo semestre de 2019. A turma se compunha de 65 estudantes matriculados. A condução da pesquisa seguiu os preceitos da Resolução do Conselho Nacional de Saúde (CNS), Resolução CNS n ${ }^{0} 466 / 12^{1}$, tal que 48 discentes anuíram em participar voluntariamente da pesquisa após lerem e assinarem um termo de consentimento livre e esclarecido, representando $73,8 \%$ da turma.

O curso de graduação em Engenharia de Software (ES) possui disciplinas que abordam os principais processos, métodos e técnicas de desenvolvimento e manutenção de software. Durante o curso de graduação, os discentes aprendem e utilizam várias ferramentas (tecnologias, software e aplicativos) nas diversas disciplinas. A IHC faz parte do quadro de disciplinas obrigatórias do currículo do curso, no quinto período, com carga horária de 4 créditos.

A disciplina IHC se desenvolve num total de 32 encontros, de 2 horas-aula cada, somando 1h5omin. Nos primeiros encontros foram apresentados a ementa, o plano da disciplina, o método de ensino, a abordagem PBL, o projeto a ser desenvolvido, o cronograma das aulas (com as datas do planejamento do projeto avaliativo, de cada conteúdo a ser ministrado, das aulas de tutoria, de todas as apresentações parciais e final do projeto e da avaliação da disciplina) e a proposta de divisão dos grupos. O projeto previa encontrar um Website com pouca usabilidade para aplicar os conceitos da disciplina IHC com o fito de melhorá-lo nesse quesito.

No quarto encontro, cada grupo apresentava os integrantes, o projeto a ser desenvolvido na disciplina com o contexto da aplicação, o planejamento (incluindo o cronograma com as datas de entrega dos artefatos a serem desenvolvidos, das apresentações parciais e da apresentação final do projeto) e as ferramentas a serem utilizadas. O cronograma do projeto deveria seguir o cronograma da disciplina constante do plano de ensino. Do quinto até o $28^{\circ}$ encontro, a dinâmica de ensino e aprendizagem foi distribuída nos seguintes blocos: aulas de conceitos, aulas de tutoria e aulas para as apresentações.

A depender dos conceitos, poderia ser ministrado um ou dois conceitos juntos em um ou dois encontros. Na aula de tutoria, cada grupo apresentava as dúvidas sobre a aplicação do conteúdo ministrado no projeto, uma espécie de sessão de tutoria, que, no geral, constituía um encontro. Na aula para as apresentações, o docente fazia avaliações parciais do andamento do projeto.

Os critérios de avaliação adotados foram quatro: (i) colaboração (participação nas atividades do projeto): o ideal era que cada membro colaborasse um pouco com cada tipo de atividade para ter um aprendizado mais abrangente no projeto; (ii) pontualidade (entrega das atividades nos prazos estabelecidos); (iii) proatividade (iniciativa e interesse na participação do projeto): os discentes deveriam requisitar tarefas e se responsabilizar por elas; e (iv) qualidade do trabalho entregue: se não exigia retrabalho e se a qualidade correspondia ao nível de exigência estabelecido.

A aula das apresentações poderia tomar até dois encontros, em que cada grupo expunha os resultados da aplicação do conteúdo no projeto para o professor e para os demais

${ }^{1}$ Disponível em: https://conselho.saude.gov.br/resolucoes/2012/Reso466.pdf 
grupos da turma, compartilhando os resultados e as soluções, permitindo que todos apresentassem dúvidas e/ou colocações sobre o conteúdo, além de conferir o retorno das contribuições e as dificuldades de aplicá-lo nos projetos.

Os grupos deveriam utilizar ferramentas para comunicação (troca de mensagens e videoconferência), para planejar e gerenciar as atividades, e para armazenar arquivos de forma colaborativa. Foi solicitado apenas o compartilhamento dos artefatos do projeto em uma Wiki no repositório da disciplina (https://github.com/Interacao-HumanoComputador), enquanto as demais ferramentas deveriam ser definidas em grupo, o que motivou a realização dessa pesquisa sobre os critérios de escolha e sobre a interação dos integrantes com elas.

Os dados foram coletados por meio da aplicação de um questionário impresso aos discentes da disciplina. Com questões abertas, fechadas de múltipla escolha e algumas questões fechadas utilizando a escala Likert, o questionário tinha o objetivo de identificar as principais tecnologias sociais e avaliar o grau de utilização, grau de conhecimento e dificuldade das tecnologias sociais utilizadas pelos discentes.

$\mathrm{O}$ primeiro bloco do questionário se relacionava às questões para identificar o perfil dos discentes, enquanto os demais blocos tratavam sobre a interação das ferramentas digitais utilizadas no cotidiano da universidade. O primeiro bloco, sobre o perfil dos respondentes, abordou perguntas sobre a idade, o sexo, qual semestre o discente está cursando, a forma de ingresso na universidade, em qual ou quais atividades deseja atuar após concluir o curso, qual ou quais dispositivos eletrônicos utiliza nas atividades de estudo e quais recursos são utilizados para buscar mais conhecimentos nas disciplinas. O segundo bloco continha perguntas sobre as ferramentas de comunicação para avisos gerais (marcar reunião e outros) e ferramentas de comunicação para realizar reunião a distância e o grau de interação dos discentes com elas; o terceiro bloco, perguntas sobre as ferramentas utilizadas no planejamento e gerenciamento das atividades e o grau de interação com elas. Já o quarto e último bloco contemplava perguntas para identificar as ferramentas para armazenar arquivos de forma colaborativa e o grau de interação com elas.

Quanto às variáveis demográficas, este era o perfil dos respondentes: 42 homens $(87,5 \%)$ e 6 mulheres $(12,5 \%)$, na faixa etária entre 19 e 36 anos, a maioria $(77,1 \%)$ na idade entre 20 e 22 anos. Os respondentes cursaram a disciplina entre o quinto e o nono semestre do curso de Engenharia de Software, sendo a maioria (62,5\%) do 50 semestre.

Esses dados foram tabulados, deles extraindo-se inferências com base na sua análise. Todos os valores percentuais da pesquisa estão arredondados em uma casa decimal após a vírgula.

\section{Discussão dos Resultados}

Esta seção divide-se em duas subseções. A primeira apresenta os resultados obtidos nas respostas ao questionário sobre o perfil, enquanto a segunda confere detalhes sobre a avaliação do uso de tecnologias sociais na aprendizagem. 


\subsection{Perfil, Interesses dos Discentes e Dispositivos Utilizados nas Atividades de Estudos}

Os dados coletados com os respondentes revelaram as seguintes formas de ingresso na Universidade: 39,6\% ingressaram pelo Programa de Avaliação Seriada (PAS), 45,8\% pelo Vestibular Tradicional, 12,5\% pelo Sistema de Seleção Unificada do Ministério da Educação (SiSU/MEC) e 2,1\% por Mobilidade Acadêmica. Os demais dados coletados sobre o perfil deles foram as áreas em que pretendiam atuar depois de formados, quais os dispositivos utilizados nas atividades de estudo e quais os recursos utilizados para buscar mais conhecimento na disciplina.

A Tabela 1 traz a percentagem das áreas em que os respondentes pretendem atuar após a conclusão do curso, predominando a área de "Desenvolvimento de Software" com maior interesse de atuação, com 70,8\%. Outra área de atuação almejada é a de "Gerente de Equipe de Desenvolvimento de Software", com 43,8\%, seguida da área de desenvolvimento de "Aplicativos para Dispositivos Móveis”, com 39,6\%.

A área de Administrador de Banco de Dados foi a que despertou menor interesse nos respondentes. A opção "Outra atividade" recebeu 10,4\%. Nesse item, foram mencionadas outras áreas como Desenvolvedor de Front-end, Marketing, Especialista em Inteligência Artificial, Machine Learning, Engenheiro DevOps, Perícia Digital.

\begin{tabular}{lc}
\hline Área de Atuação & Percentual de Discentes \\
\hline Desenvolvimento de Software & $70,8 \%$ \\
\hline Gerente de Equipe de Software & $43,8 \%$ \\
\hline Aplicativos para Dispositivos Móveis & $39,6 \%$ \\
\hline Cientista de Dados & $27,9 \%$ \\
\hline Engenheiro de Dados & $27,9 \%$ \\
\hline Empreendedor (Startup) & $27,1 \%$ \\
\hline Desenvolvimento de Jogos & $25 \%$ \\
\hline Arquiteto de Software & $25 \%$ \\
\hline Funcionário Público & $12,5 \%$ \\
\hline Outra Atividade & $10,4 \%$ \\
\hline Administrador de Banco de Dados & $6,3 \%$
\end{tabular}

Tabela 1 - Áreas de atuação desejadas após a conclusão do curso de graduação

Quanto aos dispositivos utilizados nas atividades de estudo, os resultados podem ser vistos na Tabela 2, que mostra o Notebook como o dispositivo mais utilizado por eles, com 95,8\%, seguido pelo Smartphone, com 72,9\%, depois o Computador Desktop, com $31,1 \%$, e o Tablet com 6,3\%. A opção Netbook não recebeu nenhum voto. 


\begin{tabular}{lc}
\hline Dispositivo & Percentual de Discentes \\
\hline Notebook & $95,8 \%$ \\
\hline Smartphone & $72,9 \%$ \\
\hline Computador Desktop & $31,1 \%$ \\
\hline Tablet & $6,3 \%$ \\
\hline Netbook & $0 \%$ \\
\hline
\end{tabular}

Tabela 2 - Dispositivos usados nas atividades de estudo

Na Tabela 3, os recursos utilizados para busca de mais conhecimento nas disciplinas. O Material disponibilizado pelo professor em meio digital e a Wiki de trabalhos de discentes de semestres anteriores foram os recursos mais utilizados para busca de mais conhecimento nas disciplinas pelos discentes, com 68,8\%. Em diversas disciplinas do curso de Engenharia de Software da UnB as atividades são entregues aos professores por meio de uma plataforma de ensino da instituição, chamada aprender.unb.br, ou por meio de wikis dos próprios discentes, que eles podem usar como exemplos nos semestres posteriores. A Wikipedia e os Websites de empresas especializadas, que atuam na área da disciplina ou do curso de Engenharia de Software, foram os recursos menos utilizados para buscar mais conhecimento nas disciplinas, com 18,8\% e 12,5\%, respetivamente.

\begin{tabular}{ll}
\hline Recurso & Percentual de Discentes \\
\hline Material disponibilizado pelo professor em meio digital & $68,8 \%$ \\
\hline Wiki de trabalhos de outros discentes de semestres anteriores & $68,8 \%$ \\
\hline Websites de pesquisas & $66,7 \%$ \\
\hline YouTube & $66,7 \%$ \\
\hline Videoaulas & $64,6 \%$ \\
\hline Livro digital & $54,2 \%$ \\
\hline Artigos científicos & $45,8 \%$ \\
\hline Websites educacionais & $45,8 \%$ \\
\hline Wiki de trabalhos de outros discentes da mesma turma & $33,3 \%$ \\
\hline Livro físico & $22,9 \%$ \\
\hline Wikipedia & $18,8 \%$ \\
\hline Websites de empresas especializadas & $12,5 \%$ \\
\hline
\end{tabular}

Tabela 3 - Recursos utilizados em busca de mais conhecimento nas disciplinas

\subsection{Avaliação do Uso de Tecnologias Digitais Sociais no Processo Colaborativo de Ensino e Aprendizagem pelos Discentes}

Nesta subseção apresenta-se o grau de interação com as tecnologias, cujos resultados são divididos em três partes: a primeira sobre as ferramentas de comunicação para avisos gerais (marcar reunião e outros) e ferramentas de comunicação sobre reunião a distância; a segunda sobre as ferramentas utilizadas no planejamento e gerenciamento 
das atividades; e a última, as perguntas para identificar as ferramentas para armazenar arquivos de forma colaborativa para o trabalho em equipe.

\section{- Ferramentas de Comunicação}

Na coleta de dados referentes ao uso de ferramentas para avisos gerais e agendar reunião, eles marcavam as que utilizavam. Para cada ferramenta selecionada podiam atribuir nota de 1 a 5 (sendo 1 a nota mínima e 5 a máxima) para o grau de utilização, para o grau de conhecimento e para o grau de dificuldade para utilizá-la.

A Tabela 4 mostra que o Telegram é o aplicativo mais utilizado, com 47 respondentes (97,9\%). O Telegram recebeu nota média de 4,6 com desvio padrão de o,6 para o grau de utilização, e nota média de 4,4 com desvio padrão de o,7 para o grau de conhecimento sobre o aplicativo. A dificuldade de utilização recebeu nota média 1,3 com desvio padrão de 0,9.

\begin{tabular}{|c|c|c|c|c|c|c|c|c|c|}
\hline \multirow[b]{2}{*}{ Ferramenta } & \multirow{2}{*}{$\begin{array}{l}\text { No de } \\
\text { respon- } \\
\text { dentes }\end{array}$} & \multirow[b]{2}{*}{ Interação } & \multicolumn{5}{|c|}{ Frequência Relativa } & \multirow[b]{2}{*}{ 荧 } & \multirow{2}{*}{ ช゚ } \\
\hline & & & $\frac{5}{\frac{\pi}{2}}$ & $\frac{N}{\frac{0}{\pi}}$ & $\frac{n}{3}$ & $\frac{\dot{\pi}}{\frac{0}{\pi}}$ & $\frac{0}{\frac{0}{\pi}}$ & & \\
\hline \multirow{3}{*}{ Telegram } & \multirow{3}{*}{$\begin{array}{l}47 \\
(97,9 \%)\end{array}$} & Utilização & $\mathrm{O} \%$ & $0 \%$ & $8,5 \%$ & $21,3 \%$ & $70,2 \%$ & 4,6 & 0,6 \\
\hline & & $\begin{array}{l}\text { Conhecimento } \\
\text { (expertise) }\end{array}$ & $\mathrm{O} \%$ & $\mathrm{O} \%$ & $12,8 \%$ & $36,2 \%$ & $51,1 \%$ & 4,4 & 0,7 \\
\hline & & $\begin{array}{l}\text { Dificuldade no } \\
\text { uso }\end{array}$ & $83 \%$ & $8,5 \%$ & $4,3 \%$ & o\% & $4,3 \%$ & 1,3 & 0,9 \\
\hline \multirow{3}{*}{ Whatsapp } & \multirow{3}{*}{$\begin{array}{l}33 \\
(68,8 \%)\end{array}$} & Utilização & $3 \%$ & $9,1 \%$ & $6,1 \%$ & $3 \%$ & $78,8 \%$ & 4,5 & 1,1 \\
\hline & & $\begin{array}{l}\text { Conhecimento } \\
\text { (expertise) }\end{array}$ & O\% & $3 \%$ & $\mathrm{O} \%$ & $18,2 \%$ & $78,8 \%$ & 4,7 & 0,6 \\
\hline & & $\begin{array}{l}\text { Dificuldade no } \\
\text { uso }\end{array}$ & $90,9 \%$ & $6,1 \%$ & O\% & o\% & $3 \%$ & 1,2 & 0,7 \\
\hline \multirow{3}{*}{ Wire } & \multirow{3}{*}{$\begin{array}{l}5 \\
(10,4 \%)\end{array}$} & Utilização & $20 \%$ & $20 \%$ & $40 \%$ & $\mathrm{O} \%$ & $0 \%$ & 2,8 & 1,3 \\
\hline & & $\begin{array}{l}\text { Conhecimento } \\
\text { (expertise) }\end{array}$ & $40 \%$ & $20 \%$ & $20 \%$ & $20 \%$ & O\% & 2,2 & 1,2 \\
\hline & & $\begin{array}{l}\text { Dificuldade no } \\
\text { uso }\end{array}$ & $60 \%$ & $40 \%$ & O\% & o\% & O\% & 1,4 & 0,5 \\
\hline \multirow{3}{*}{ Slack } & \multirow{3}{*}{$\begin{array}{l}5 \\
(10,4 \%)\end{array}$} & Utilização & $\mathrm{O} \%$ & $\mathrm{O} \%$ & $60 \%$ & $20 \%$ & $20 \%$ & 3,6 & 0,8 \\
\hline & & $\begin{array}{l}\text { Conhecimento } \\
\text { (expertise) }\end{array}$ & o\% & $20 \%$ & $40 \%$ & $20 \%$ & $20 \%$ & 3,4 & 1 \\
\hline & & $\begin{array}{l}\text { Dificuldade no } \\
\text { uso }\end{array}$ & $40 \%$ & $20 \%$ & $40 \%$ & o\% & O\% & 2 & 0,9 \\
\hline \multirow{3}{*}{$S M S$} & \multirow{3}{*}{$\begin{array}{l}4 \\
(8,3 \%)\end{array}$} & Utilização & $50 \%$ & $25 \%$ & $0 \%$ & $0 \%$ & $25 \%$ & 2,3 & 1,6 \\
\hline & & $\begin{array}{l}\text { Conhecimento } \\
\text { (expertise) }\end{array}$ & o\% & o\% & O\% & O\% & $75 \%$ & 5 & $\mathrm{O}$ \\
\hline & & $\begin{array}{l}\text { Dificuldade no } \\
\text { uso }\end{array}$ & $75 \%$ & o\% & O\% & O\% & O\% & 1 & $\mathrm{O}$ \\
\hline
\end{tabular}

Tabela 4 - Ferramentas para avisos gerais e agendamento de reuniões 
A segunda ferramenta para avisos gerais e marcar reunião mais utilizada foi o Whatsapp, com 33 discentes (68,8\%), média de 4,5 e desvio padrão de 1,1; para o grau de utilização, média 4,7 com desvio padrão o,6 para o grau de conhecimento da ferramenta; a média do grau de dificuldade para utilizar o aplicativo foi de 1,2 com desvio padrão de 0,7.

Após a análise da preferência dessas ferramentas, alguns discentes da turma foram presencialmente questionados sobre a razão dessa preferência pelo Telegram; as razões citadas foram que para falar com uma pessoa pelo Telegram não precisa do número do telefone dela, é necessário apenas o @; a possibilidade de fazer enquetes de votação dentro dos grupos, o de número de pessoas permitido no grupo; stickers mais bonitos; possibilidade de usar o Telegram pelo computador sem que o aparelho celular esteja por perto; e usar chatbot do aplicativo para controle de projetos.

Sobre a preferência das ferramentas para realizar reunião a distância, a primeira pergunta foi sobre o número de pessoas que participavam das reuniões com uso dessas ferramentas. Eles responderam que as usam para reuniões de 2 até 10 pessoas; a maioria dos respondentes usa para reuniões de 6 pessoas $(45,8 \%)$, seguida de reuniões com 5 pessoas $(29,2 \%)$.

A título de ilustração, seguem algumas respostas dos discentes: “Acho que o Telegram é uma ferramenta muito boa para a comunicação entre os discentes, eu me sinto mais livre dentro do Telegram."; e "Para usar na faculdade, no trabalho, em grupos de estudo ou de pesquisa eu prefiro o Telegram por suas funcionalidades adicionais. Agora, para usar num contato pessoal, com pessoas mais íntimas, ou de um modo mais 'relaxado', prefiro o WhatsApp."

Em seguida, eles responderam sobre as ferramentas usadas para trocar mensagens instantâneas e/ou para videoconferências nas reuniões (Tabela 5), em que predomina o Google hangouts. Às perguntas relacionadas com a interação com essas ferramentas, os discentes atribuíram notas de 1 a 5 para o grau de utilização, para o grau de conhecimento da ferramenta e para grau de dificuldade para utilizá-la. Os resultados podem ser vistos na Tabela 6.

\begin{tabular}{lccc}
\hline Ferramenta & Discentes & Videoconferência & Mensagens instantâneas \\
\hline Google hangouts & $44(91,7 \%)$ & $44(91,7 \%)$ & $2(4,2 \%)$ \\
\hline Discord & $19(39,6 \%)$ & $16(33,3 \%)$ & $8(16,7 \%)$ \\
\hline Skype & $4(8,3 \%)$ & $4(8,3 \%)$ & $0 \%$ \\
\hline TeamSpeak & $1(2,1 \%)$ & $1(2,1 \%)$ & $0 \%$ \\
\hline
\end{tabular}

Tabela 5 - Ferramentas para trocar mensagem e videoconferência

A Tabela 6 evidencia que o Google hangouts é a ferramenta mais utilizada, com 44 discentes (91,7\%). Recebeu nota média de 4,1 com desvio padrão de 0,9 para o grau de utilização e nota média de 4,2 com desvio padrão de o,8 para o grau de conhecimento sobre a ferramenta. Sobre a dificuldade de utilizá-la, os respondentes atribuíram nota média de 1,6 com desvio padrão de 0,9. A segunda mais utilizada foi o Discord, com 19 discentes (39,6\%). Este recebeu média 4 com desvio padrão de 1 para o grau de uso; 
para o grau de conhecimento da ferramenta, média 4,7 com desvio padrão o,6; e o grau de dificuldade para utilizar o aplicativo foi de 1,8 com desvio padrão de 1,2. As demais ferramentas mais utilizadas citadas foram o Skype seguido pelo TeamSpeak, com 4 discentes $(8,3 \%)$ e $1(2,1 \%)$, respectivamente.

\begin{tabular}{|c|c|c|c|c|c|c|c|c|c|}
\hline \multirow[b]{2}{*}{ Ferramenta } & \multirow{2}{*}{$\begin{array}{l}\text { No de } \\
\text { respon- } \\
\text { dentes }\end{array}$} & \multirow[b]{2}{*}{ Interação } & \multicolumn{5}{|c|}{ Frequência Relativa } & \multirow[b]{2}{*}{ 冚 } & \multirow{2}{*}{ 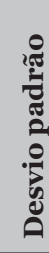 } \\
\hline & & & है & $\frac{N}{\dot{0}}$ & $\frac{\infty}{\stackrel{0}{\sigma}}$ & $\stackrel{\dot{\partial}}{\frac{\dot{\theta}}{\pi}}$ & $\stackrel{10}{\frac{a}{\pi}}$ & & \\
\hline \multirow{3}{*}{$\begin{array}{l}\text { Google } \\
\text { hangouts }\end{array}$} & \multirow{3}{*}{$\begin{array}{c}44 \\
(91,7 \%)\end{array}$} & Utilização & $0 \%$ & $4,6 \%$ & $20,5 \%$ & $31,8 \%$ & $40,9 \%$ & 4,1 & 0,9 \\
\hline & & $\begin{array}{l}\text { Conhecimento } \\
\text { (expertise) }\end{array}$ & $0 \%$ & $2,3 \%$ & $18,2 \%$ & $36,4 \%$ & $40,9 \%$ & 4,2 & 0,8 \\
\hline & & $\begin{array}{l}\text { Dificuldade no } \\
\text { uso }\end{array}$ & $61,4 \%$ & $22,7 \%$ & $6,8 \%$ & $6,8 \%$ & $0 \%$ & 1,6 & 0,9 \\
\hline \multirow{3}{*}{ Discord } & \multirow{3}{*}{$\begin{array}{c}19 \\
(39,6 \%)\end{array}$} & Utilização & $0 \%$ & $10,5 \%$ & $21,1 \%$ & $26,3 \%$ & $42,1 \%$ & 4 & 1 \\
\hline & & $\begin{array}{l}\text { Conhecimento } \\
\text { (expertise) }\end{array}$ & $0 \%$ & $26,3 \%$ & $15,8 \%$ & $5,3 \%$ & $52,6 \%$ & 3,8 & 1,3 \\
\hline & & $\begin{array}{l}\text { Dificuldade no } \\
\text { uso }\end{array}$ & $68,4 \%$ & $\mathrm{O} \%$ & $21,1 \%$ & $5,3 \%$ & $5,3 \%$ & 1,8 & 1,2 \\
\hline \multirow{3}{*}{ Skype } & \multirow{3}{*}{$\begin{array}{c}4 \\
(8,3 \%)\end{array}$} & Utilização & $50 \%$ & $25 \%$ & $0 \%$ & $0 \%$ & $25 \%$ & 2,3 & 1,6 \\
\hline & & $\begin{array}{l}\text { Conhecimento } \\
\text { (expertise) }\end{array}$ & $50 \%$ & $25 \%$ & O\% & $0 \%$ & $25 \%$ & 2,3 & 1,6 \\
\hline & & $\begin{array}{l}\text { Dificuldade no } \\
\text { uso }\end{array}$ & $75 \%$ & $\mathrm{O} \%$ & O\% & $25 \%$ & o\% & 1,8 & 1,3 \\
\hline \multirow{3}{*}{ TeamSpeak } & \multirow{3}{*}{$\begin{array}{c}1 \\
(2,1 \%)\end{array}$} & Utilização & o\% & $100 \%$ & $0 \%$ & $0 \%$ & o\% & 2 & o \\
\hline & & $\begin{array}{l}\text { Conhecimento } \\
\text { (expertise) }\end{array}$ & o\% & $0 \%$ & o\% & o\% & $100 \%$ & 5 & 0 \\
\hline & & $\begin{array}{l}\text { Dificuldade no } \\
\text { uso }\end{array}$ & $100 \%$ & O\% & O\% & o\% & o\% & 1 & o \\
\hline
\end{tabular}

Tabela 6 - Ferramentas para realizar reuniões

\section{- Ferramentas de planejamento e gerenciamento das atividades}

Quanto à coleta de dados sobre a preferência de ferramentas para planejar e gerenciar as atividades, para cada ferramenta selecionada os respondentes podiam atribuir uma nota de 1 a 5 para o grau de utilização, para o grau de conhecimento e para o grau de dificuldade para utilizá-la. O resultado dessas questões está na Tabela 7, que mostra que o ZenHub é a ferramenta mais utilizada, com 38 discentes (79,2\%). O ZenHub recebeu nota média de 3,2 com desvio padrão de 1,2 para o grau de utilização, e nota média de 3,2 com desvio padrão de 1,1 para o grau de conhecimento sobre a ferramenta. Na dificuldade de utilizar o aplicativo ZenHub, eles atribuíram nota média de 1,7 com desvio padrão de 0,8 . 


\begin{tabular}{|c|c|c|c|c|c|c|c|c|c|}
\hline \multirow{2}{*}{ Ferramenta } & \multirow{2}{*}{$\begin{array}{l}\text { No de } \\
\text { respon- } \\
\text { dentes }\end{array}$} & \multirow{2}{*}{ Interação } & \multicolumn{5}{|c|}{ Frequência Relativa } & \multirow[b]{2}{*}{ 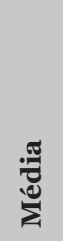 } & \multirow{2}{*}{ 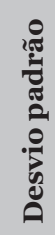 } \\
\hline & & & $\frac{5}{3}$ & 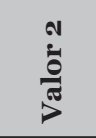 & $\begin{array}{l}\infty \\
\frac{0}{\nu} \\
\end{array}$ & $\frac{\dot{\sigma}}{\frac{0}{\pi}}$ & \begin{tabular}{l}
10 \\
$\frac{0}{\sigma}$ \\
\multicolumn{2}{c}{}
\end{tabular} & & \\
\hline \multirow{3}{*}{ ZenHub } & \multirow{3}{*}{$\begin{array}{c}38 \\
(79,2 \%)\end{array}$} & Utilização & $8,3 \%$ & $10,4 \%$ & $33,3 \%$ & $14,6 \%$ & $12,5 \%$ & 3,2 & 1,2 \\
\hline & & $\begin{array}{l}\text { Conhecimento } \\
\text { (expertise) }\end{array}$ & $6,3 \%$ & $18,8 \%$ & $16,7 \%$ & $29,2 \%$ & $8,3 \%$ & 3,2 & 1,1 \\
\hline & & $\begin{array}{l}\text { Dificuldade no } \\
\text { uso }\end{array}$ & $39,6 \%$ & $29,2 \%$ & $8,3 \%$ & $2,1 \%$ & o\% & 1,7 & 0,8 \\
\hline \multirow{3}{*}{ Trello } & \multirow{3}{*}{$\begin{array}{c}29 \\
(60,4 \%)\end{array}$} & Utilização & $14,6 \%$ & $10,4 \%$ & $16,7 \%$ & $12,5 \%$ & $6,3 \%$ & 2,8 & 1,3 \\
\hline & & $\begin{array}{l}\text { Conhecimento } \\
\text { (expertise) }\end{array}$ & $4,2 \%$ & $6,3 \%$ & $12,5 \%$ & $25 \%$ & $12, \%$ & 3,6 & 1,1 \\
\hline & & $\begin{array}{l}\text { Dificuldade no } \\
\text { uso }\end{array}$ & $47,9 \%$ & $8,3 \%$ & $2,1 \%$ & $0 \%$ & $2,1 \%$ & 1,3 & 0,8 \\
\hline \multirow{3}{*}{$\begin{array}{l}\text { Google } \\
\text { Calendar }\end{array}$} & \multirow{3}{*}{$\begin{array}{c}24 \\
(50 \%)\end{array}$} & Utilização & $10,4 \%$ & $6,3 \%$ & $20,8 \%$ & $4,2 \%$ & $8,3 \%$ & 2,9 & 1,3 \\
\hline & & $\begin{array}{l}\text { Conhecimento } \\
\text { (expertise) }\end{array}$ & $6,3 \%$ & $8,3 \%$ & $20,8 \%$ & $8,3 \%$ & $6,3 \%$ & 3 & 1,2 \\
\hline & & $\begin{array}{l}\text { Dificuldade no } \\
\text { uso }\end{array}$ & $27,1 \%$ & $18,8 \%$ & $4,2 \%$ & o\% & $0 \%$ & 1,5 & 0,6 \\
\hline \multirow{3}{*}{ Outro } & \multirow{3}{*}{$\begin{array}{c}8 \\
(16,7 \%)\end{array}$} & Utilização & o\% & $\mathrm{O} \%$ & $4,2 \%$ & $4,2 \%$ & $8,3 \%$ & 4,3 & 0,8 \\
\hline & & $\begin{array}{l}\text { Conhecimento } \\
\text { (expertise) }\end{array}$ & $0 \%$ & $0 \%$ & $4,2 \%$ & $8,3 \%$ & $4,2 \%$ & 4 & 0,7 \\
\hline & & $\begin{array}{l}\text { Dificuldade no } \\
\text { uso }\end{array}$ & $10,4 \%$ & $4,2 \%$ & $2,1 \%$ & o\% & $0 \%$ & 1,5 & 0,7 \\
\hline
\end{tabular}

Tabela 7 - Ferramentas para planejar e gerenciar as atividades

A segunda ferramenta mais utilizada foi o Trello, com 29 discentes (60,4\%), média de 3,2 e desvio padrão de 1,2 para o grau de utilização. A média 3,2 com desvio padrão 1,1 foi para o grau de conhecimento da ferramenta, e a média do grau de dificuldade para utilizar o Trello foi de 1,7 com desvio padrão de o,8. A terceira ferramenta mais citada foi o Google Calendar, por 24 discentes (50\%), com a média de 2,9 e desvio padrão de 1,3 para o grau de utilização, média 3 com desvio padrão 1,2 para o grau de conhecimento da ferramenta e a média do grau de dificuldade para utilizar o aplicativo foi de 1,5 com desvio padrão de o,6. Na opção Outro, foram citadas as seguintes ferramentas: Notion (três vezes), Google keep (duas vezes), Pipefy, Toggl, e Habitica (uma vez cada).

\section{- Ferramentas para armazenar arquivos de forma Colaborativa}

Quanto à preferência de ferramentas para armazenamento colaborativo de arquivos nas quais os integrantes do grupo poderiam armazenar e recuperar os arquivos do(s) projeto(s), eles podiam atribuir nota de 1 a 5 para o grau de utilização, para o grau de conhecimento e para o grau de dificuldade para utilizá-la. 


\begin{tabular}{|c|c|c|c|c|c|c|c|c|c|}
\hline \multirow[b]{2}{*}{ Ferramenta } & \multirow{2}{*}{$\begin{array}{l}\text { No de } \\
\text { respon- } \\
\text { dentes }\end{array}$} & \multirow[b]{2}{*}{ Interação } & \multicolumn{5}{|c|}{ Frequência Relativa } & \multirow[b]{2}{*}{ 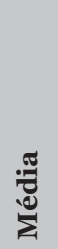 } & \multirow{2}{*}{ 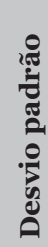 } \\
\hline & & & $\stackrel{5}{\frac{0}{\pi}}^{-1}$ & $\frac{N}{\frac{0}{2}}$ & $\stackrel{n}{\frac{0}{\pi}}$ & $\frac{+}{\stackrel{0}{0}}$ & $\frac{10}{\pi}$ & & \\
\hline \multirow{3}{*}{ Google Drive } & \multirow{3}{*}{$\begin{array}{c}48 \\
(100 \%)\end{array}$} & Utilização & O\% & O\% & $6,3 \%$ & $20,8 \%$ & $72,9 \%$ & 4,7 & 0,6 \\
\hline & & $\begin{array}{l}\text { Conhecimento } \\
\text { (expertise) }\end{array}$ & $2,1 \%$ & $2,1 \%$ & $20,8 \%$ & $29,2 \%$ & $45,8 \%$ & 4,1 & 1 \\
\hline & & $\begin{array}{l}\text { Dificuldade no } \\
\text { uso }\end{array}$ & $77,1 \%$ & $18,8 \%$ & o\% & $2,1 \%$ & $2,1 \%$ & 1,3 & 0,8 \\
\hline \multirow{3}{*}{ Dropbox } & \multirow{3}{*}{$\begin{array}{c}4 \\
(8,3 \%)\end{array}$} & Utilização & $25 \%$ & $\mathrm{O} \%$ & $25 \%$ & $\mathrm{O} \%$ & $50 \%$ & 3,5 & 1,7 \\
\hline & & $\begin{array}{l}\text { Conhecimento } \\
\text { (expertise) }\end{array}$ & o\% & O\% & O\% & $25 \%$ & $50 \%$ & 4 & 1,2 \\
\hline & & $\begin{array}{l}\text { Dificuldade no } \\
\text { uso }\end{array}$ & $75 \%$ & $25 \%$ & O\% & O\% & O\% & 1,3 & 0,4 \\
\hline
\end{tabular}

Tabela 8 - Ferramentas para armazenar arquivos de forma colaborativa

Os discentes citaram uso de apenas duas ferramentas (Google Drive e Dropbox), como se vê na Tabela 8. O Google Drive é a ferramenta utilizada por todos os 48 discentes (100\%), recebendo nota média de 4,7 com desvio padrão de o,6 para o grau de utilização e nota média de 4,1 com desvio padrão de 1 para o grau de conhecimento sobre a ferramenta. Sobre a dificuldade de utilizar o Google Drive, eles atribuíram nota média de 1,3 com desvio padrão de 0,8 . O Dropbox é utilizado por 4 discentes $(8,3 \%)$, recebendo média de 3,5 com desvio padrão de 1,7 para o grau de utilização.

\section{- Ferramentas para Repositório de artefatos}

Quanto à preferência de ferramentas para controle de versão dos artefatos (usados para controlar as diferentes versões, histórico e desenvolvimento, códigos-fonte e documentação), para cada ferramenta selecionada, os respondentes podiam atribuir nota de 1 a 5 para o grau de utilização, para o grau de conhecimento e para o grau de dificuldade no uso da ferramenta selecionada. Eles utilizam duas ferramentas (GitHub e GitLab), como ser vê na Tabela 9.

O GitHub é a ferramenta mais utilizada, por 46 discentes (95,8\%); recebeu nota média de 4,8 com desvio padrão de o,4 para o grau de utilização e nota média de 3,9 com desvio padrão de o,8 para o grau de conhecimento sobre a ferramenta. Sobre a dificuldade de seu uso, eles atribuíram nota média de 1,6 com desvio padrão de o,8. O GitLab é a segunda ferramenta mais utilizada, por 16 discentes (33,3\%), com a média de 2,9 com desvio padrão de 1,3 para o grau de utilização. 


\begin{tabular}{|c|c|c|c|c|c|c|c|c|c|}
\hline \multirow[b]{2}{*}{ Ferramenta } & \multirow{2}{*}{$\begin{array}{l}N^{\circ} \text { de } \\
\text { respon- } \\
\text { dentes }\end{array}$} & \multirow[b]{2}{*}{ Interação } & \multicolumn{5}{|c|}{ Frequência Relativa } & \multirow[b]{2}{*}{ 㞼 } & \multirow{2}{*}{ 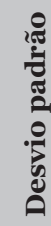 } \\
\hline & & & $\frac{\overrightarrow{0}}{\stackrel{0}{\circ}}$ & 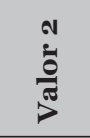 & $\begin{array}{l}\infty \\
\stackrel{0}{\sigma} \\
\stackrel{0}{\circ}\end{array}$ & $\frac{\dot{\sigma}}{\frac{0}{\pi}}$ & $\frac{10}{\stackrel{0}{\sigma}}$ & & \\
\hline \multirow{3}{*}{ GitHub } & \multirow{3}{*}{$\begin{array}{c}46 \\
(95,8 \%)\end{array}$} & Utilização & o\% & $0 \%$ & $0 \%$ & $17,4 \%$ & $82,6 \%$ & 4,8 & 0,4 \\
\hline & & $\begin{array}{l}\text { Conhecimento } \\
\text { (expertise) }\end{array}$ & O\% & $4,3 \%$ & $26,1 \%$ & $45,7 \%$ & $23,9 \%$ & 3,9 & 0,8 \\
\hline & & $\begin{array}{l}\text { Dificuldade no } \\
\text { uso }\end{array}$ & $56,4 \%$ & $26,1 \%$ & $15,2 \%$ & $2,2 \%$ & O\% & 1,6 & 0,8 \\
\hline \multirow{3}{*}{ GitLab } & \multirow{3}{*}{$\begin{array}{c}16 \\
(33,3 \%)\end{array}$} & Utilização & $12,5 \%$ & $31,3 \%$ & $31,3 \%$ & $6,3 \%$ & $18,8 \%$ & 2,9 & 1,3 \\
\hline & & $\begin{array}{l}\text { Conhecimento } \\
\text { (expertise) }\end{array}$ & $18,8 \%$ & $6,3 \%$ & $25 \%$ & $31,3 \%$ & $12,5 \%$ & 3,1 & 1,3 \\
\hline & & Dificuldade no & $50 \%$ & $25 \%$ & $6,3 \%$ & $6,3 \%$ & $6,3 \%$ & 1,9 & 1,2 \\
\hline
\end{tabular}

Tabela 9 - Ferramentas para repositórios de artefatos

Algumas particularidades da aplicação dessa abordagem na disciplina:

- número de estudantes envolvidos, acima de 60 (sessenta);

- heterogeneidade no perfil discente, predominando estudantes do quinto período do curso; e

- $\quad$ uso de práticas complementares visando a mitigar problemas identificados na Aprendizagem baseada em Projetos. Cabe mencionar, dentre essas práticas complementares, o uso de atividades para incentivar iniciativas com entregas individuais periódicas, dentro ou fora do escopo do projeto.

\section{Considerações Finais}

As tecnologias digitais sociais podem auxiliar na aprendizagem colaborativa, que é regida pelas novas relações com o saber, motivando a interagir com as próprias tecnologias, e podem ser aplicadas de forma inovadora no ensino superior, quando aliadas a novas metodologias de ensino. Essas metodologias, no entanto, devem considerar as novas relações com o saber e com a interação social, mediada ou não por tecnologia.

Este artigo apresentou e discutiu os resultados da abordagem de metodologia ativa orientada a projetos na disciplina de IHC do curso superior em Engenharia de Software de uma instituição federal, que pode ser replicado em outras realidades, dessa área de conhecimento ou não, demonstrando que as ferramentas digitais sociais são adequadas no suporte ao trabalho colaborativo em todas as fases de construção do conhecimento num processo colaborativo de ensino e aprendizagem. Dos 65 estudantes matriculados, que formaram 10 grupos de 6 estudantes e um grupo com 5, teve como resultado a aprovação de 64 estudantes que concluíram a disciplina, mostrando que a metodologia 
adotada, baseada em projetos, foi adequada aos objetivos pedagógicos traçados pelo docente em seu planejamento.

Diferentes ferramentas foram eleitas pelos estudantes, para diferentes tarefas, motivadas pela percepção de cada um sobre usabilidade e adequação a cada uma das tarefas necessárias à construção do projeto. Essas tecnologias digitais, consideradas aqui como sociais pela interação promovida entre os estudantes, foram fundamentais no suporte, armazenamento e gerenciamento de todo o processo de ensino e aprendizagem colaborativamente construído.

Os resultados desta pesquisa revelam que as tecnologias digitais sociais mais utilizadas foram: para comunicação foi o Telegram; para videoconferências foi o Google hangouts; para planejamento e gerenciamento das atividades do projeto foi o ZenHub; para armazenamento colaborativo de arquivos foi o Google Drive; e para controle de versão dos artefatos foi o GitHub.

Como trabalhos futuros, e já em andamento, encontram-se: o planejamento da aplicação da abordagem nesta e noutras disciplinas do curso de Engenharia de Software da UnB e em disciplinas do curso de Ciência da Computação na Universidade Estadual do Oeste do Paraná - UNIOESTE, para identificar restrições da aplicação da abordagem e seu aprimoramento contínuo, além do planejamento da aplicação desta pesquisa sobre o conhecimento e comparação das tecnologias digitais sociais utilizadas em outras turmas e em uma universidade de outra região do Brasil, com estudantes com perfis diferentes.

\section{Referências}

Bender W. N. (2014). Aprendizagem Baseada em Projetos: Educação diferenciada para o século XXI. Porto Alegre: Penso Editora.

Blumenfeld, P. C. (1991). Motivating project-based learning: Sustaining the doing, supporting the learning. Educational Psychologist, 26(3-4), 369-398.

Cerutti, D. M. L. (2010) Ensino de IHC: Desconstruindo interfaces em sala de aula. In WEIHC - Workshop sobre Ensino de IHC. Belo Horizonte, MG.

Chagas, A. M., Linhares, R. N., \& Mota, M. F. (2019). A Curadoria de Conteúdo Digital enquanto Proposta Metodológica e Multirreferencial. RISTI - Revista Ibérica de Sistemas e Tecnologias de Informação, (33), 32-47. doi: http://dx.doi.org/10.17013/ risti.33.32-47.

Correia, W. C. C., \& Oliveira, G. F. (2020). Reflexões sobre a prática da interdisciplinaridade através da metodologia Project Based Learning: Um estudo de caso no ensino de Engenharia. Revista Docência do Ensino Superior, 10. doi: https://doi.org/10.35699/2237-5864.2020.13597.

Fernàndez March, A. (2006). Metodologías activas para la formación de competencias. Educatio siglo XXI, Revista de la Facultad de Ciencias de la Educación de la Universidad de Murcia, 24, 35-56. 
Figueiredo R. M., Sales, A. B., Ribeiro, L. C. M., Laranjeira, L. A. F. and Rocha, A., (2010). "Teaching Software Quality in an Interdisciplinary Course of Engineering," In: 7th International Confer. on the Quality of Information and Communications Technology (QUATIC), pp. 144-149. https://doi.org/10.1109/QUATIC.2010.28.

Filatro, A., \& Cavalcanti, C. C. (2018). Metodologias Inov-ativas na educação presencial, a distância e corporativa (1th Ed.). São Paulo: Saraiva Educação.

Gerhardt, T., \& Silveira, D. T. (2009). Métodos de pesquisa. Porto Alegre: Editora da UFRGS.

Gibbs, W. (1994). Software’s chronic crisis. Scientific American, 271 (3), 86-95.

Gil, A. C. (1999). Métodos e Técnicas de Pesquisa Social (5th Ed.). São Paulo: Atlas.

Instituto Brasileiro de Geografia e Estatística. (2018). Pesquisa nacional por amostra de domicílios: Acesso à internet e à televisão e posse de telefone móvel celular para uso pessoal: 2017. Disponível em: https://biblioteca.ibge.gov.br/index.php/bibliotecacatalogo ?view=detalhes\&id $=\mathbf{2 1 0 1 6 3 1}$.

Junior, P. T. A. (2010). Papéis do Docente em IHC: do conhecimento ao mercado. In Anais Extendidos do IX Simpósio de Fatores Humanos em Sistemas Computacionais (Volume II). Porto Alegre, RS: Sociedade Brasileira de Computação.

Lima, J. V., Júnior, M. de M. A., Moya, A., Almeida, R., Anjos, P., Lencastre, M., Fagundes, R. A. de A., \& Alencar, F. (2019). As Metodologias Ativas e o Ensino em Engenharia de Software: uma revisão sistemática da literatura. In Proceedings of VIII Congresso Brasileiro de Informática na Educação (CBIE), Anais do XXV Workshop de Informática na Escola (WIE), Brasília, Brasil.

McLoughlin, C., \& Lee, M. J. W. (2007). Social software and participatory learning: Pedagogical choices with technology affordances in the Web 2.0 era. In Proceedings of ASCILITE - Australian Society for Computers in Learning in Tertiary Education Annual Conference (p. 664-675). Queensland, Australia. Disponível em: https://www.learntechlib.org/p/46128/.

Sales, A. B. de, Del, A. de M., \& Sales, M. B. de. (2013). Avaliação da Aplicação da Aprendizagem Baseada em Problemas na Disciplina de Interação Humano e Computador de curso de Engenharia de Software. RENOTE, Revista Novas Tecnologias na Educação, 11(3). doi: https://doi.org/10.22456/1679-1916.44932

Santana, A. C. (2009). Metodologia para a Aplicação da Aprendizagem Orientada por Projetos (AOPj), nos Cursos de Engenharia, com Foco nas Competências Transversais. (Tese de Doutorado), Engenharia Elétrica, Universidade de Brasília, Brasília.

Stahl, G., Koschmann, T., \& Suthers, D. (2006). Computer-supported collaborative learning: An historical perspective. In: R. K. Sawyer (Ed.), Cambridge handbook of the learning sciences, (p. 409-426). Cambridge, UK: Cambridge University Press. 
Surowiecki, K. (2005). The wisdom of crowds (1st Ed.). New York, NY: Anchor Books.

Wander, C. M. P. da S., Sales, A. B. de, Santos, G. A., Hilmer, R. N., Neri, L. A. L., \& Ribeiro, L. C. M. (2012). A Elaboração da Nova Matriz Curricular do Curso de Engenharia de Software da UnB: uma proposta de Método baseado na Educação por Competências. In V Fórum de Educação em Engenharia de Software (FEES/ SBES), Natal.

Wrigley, H. S. (1998) Knowledge in action: The promise of project-based learning. Focus on Basics, 2(Dez). Disponível em: http://www.ncsall.net/index.html@ $\mathrm{id}=384 \cdot \mathrm{html} /$ 